\title{
A spectroscopic survey of oEA stars
}

\author{
David Mkrtichian ${ }^{1 *}$ Chris Engelbrecht ${ }^{2}$, Patricia Lampens ${ }^{3}$, Holger Lehmann ${ }^{4}$, \\ Naparorn A-thano ${ }^{1}$, Khemsinan Gunsriwiwat ${ }^{1}$
}

\author{
${ }^{1}$ National Astronomical Research Institute of Thailand, Chiang Mai 50180, Thailand \\ ${ }^{2}$ Department of Physics, University of Johannesburg, PO Box 524, Auckland Park, 2006, South Africa \\ ${ }^{3}$ Koninklijke Sterrenwacht van België, Brussel 1180, Belgium \\ ${ }^{4}$ Thüringer Landessternwarte Tautenburg, Sternwarte 5, D-07778 Tautenburg, Germany
}

\begin{abstract}
We present the first results of a spectroscopic survey of southern and equatorial Algol-type eclipsing binary systems with mass-accreting pulsating components (oEA stars) using the 2.4-m Thai National Telescope (TNT) and the 10-m South African Large Telescope (SALT). The survey aims to find orbital and binary parameters and to detect the high-degree non-radial pulsations (NRPs). We report the detection of high-degree NRPs in AS Eri, WY Cet, VY Hya, GQ TrA, BD Vir, and UW Vir.
\end{abstract}

Keywords: binaries - eclipsing - stars - oscillations - oEA stars

\section{Introduction}

Mkrtichian et al. (2004) introduced the class of oEA stars. These are semi-detached eclipsing Algoltype stars with mass transfer where the A- or F-type mass-accreting primaries lie in the instability strip of the $\delta$ Scuti ( $\delta$ Sct) stars and show $\delta$ Sct-like pulsations. The unique property of oEA stars is the co-existence of oscillations with accretion. Non-stationary accretion keeps them in thermal imbalance and influences their pulsation properties and their evolution along the main sequence. Thus, in oEA stars, we can simultaneously study the relationship between tidal interaction, mass-transfer and the excitation of non-radial oscillations. Since the discovery of the class, about 72 oEA-type systems have been found via photometry (see references in Mkrtichian et al. 2018). Spectroscopic investigations of oEA pulsations have only been made for the brightest members - RZ Cas (Lehmann \& Mkrtichian 2004, 2008; Tkachenko et al. 2009), TW Dra (Tkachenko et al. 2010) and R CMa (Lehmann et al. 2018) - using 2-m class telescopes. The oEA stars are expected to be seen equatoron, which is the optimal inclination for the detection of sectoral $(\ell=m$, with $\ell$ the degree and $m$ the azimuthal number) NRPs, having their greatest amplitudes around the stellar equator. This prediction is well confirmed observationally: RZ Cas and TW Dra both show high-degree non-radial oscillations in their spectral line profile variations. From multi-site studies with a long base line, Mkrtichian et al. (2018) have found orbital period changes of the order of seconds in RZ Cas, cycling over 6-9 years, correlated with pulsation amplitude and frequency variability. They have shown that the variations are caused by transient phases of magnetic activity on the Roche lobe filling component (Applegate's

*davidmkrt@gmail.com 
hypothesis) and that the correlated variations of pulsations are caused by high mass-transfer episodes. These mass-transfer episodes accrete additional angular momentum onto the surface of the pulsating star, forcing modal amplitude variations and rotational acceleration. The latter is detected through the Doppler effect. The pulsation frequency variations which measure the acceleration or the braking of surface layers are $m$-dependent, permitting a precise measure (for high-degree modes) of the rotational variations. This dependence also facilitates the identification of the pulsation modes. Thus, the detection, identification and precise monitoring of frequencies of high-degree NRPs are crucial tools for these studies.

\section{The spectroscopic survey}

Only a few bright oEA stars have been studied spectroscopically for pulsational analyses. The purpose of this project is to extend the spectroscopic studies to fainter northern and southern oEA stars, in order to investigate the interaction of mass transfer, pulsation and short-term dynamical evolution of these binary systems in detail. Thanks to the technique of Doppler imaging of the rotationally broadened spectral line profiles, spectroscopy is more sensitive than photometry for the detection of high-degree NRPs, which is why we are gathering spectroscopy with 2-m and 10-m class telescopes for this project.

The spectroscopic survey of oEA stars has been started in 2014 using the 2.4-m Thai National Telescope at the Thai National Observatory (TNO) in Thailand. Spectra were acquired with a fiberfed medium-resolution $(\mathrm{R}=19000)$ echelle spectrometer MRES.

During the first workshop of the Belgo-Indian Network for Astronomy and astrophysics (BINA) in November 2016 (De Cat et al. 2018), the first two authors of this article agreed to cooperate and expand the spectroscopic survey to the oEA stars in the southern hemisphere. In 2017, the survey was continued using the SALT dual-beam fiber-fed echelle spectrograph HRS $(\mathrm{R}=40000)$ (Bramall et al., 2012) at the South African Observatory (SAAO) in Sutherland (South Africa). Spectroscopy of some oEA systems were also carried out using the High-Efficiency and high-Resolution Mercator Echelle Spectrograph (HERMES; Raskin et al. 2011) at the 1.2-m Mercator telescope at Roque de los Muchachos Observatory (RMO, Spain) and using the high-resolution $(\mathrm{R}=30000)$ Coude-Echelle spectrograph attached to the 2.0-m Alfred Jensch Telescope at the Thüringer Landessternwarte Tautenburg (TLS, Germany).

Firstly, using the HRS and MRES, we obtained spectroscopic time-series observations by following a target star for an entire track (i.e. for 45 to 180 minutes) for HRS or during longer intervals of nights for the MRES. Using this mode of sequential observations we obtained a sufficient time sampling of the spectral line profiles per pulsation cycle to identify the pulsation modes. Secondly, using these blocks of medium- and high-resolution time-series spectroscopy, spread over many nights (= different orbital phases), we aimed to improve the modal frequency estimations, to get precise radial velocity orbits for these oEA systems and to derive precise orbital elements and absolute physical parameters. Thirdly, from the orbital phase-distributed high-resolution spectra survey we aimed to determine the chemical composition, effective temperature $\mathrm{T}_{\text {eff }}$, surface gravity $\log g$, and projected rotational velocity $v \sin i$ for both components. Using the profiles of $\mathrm{H} \alpha$ and $\mathrm{He}$ I line we may see spectroscopic signs of non-stationary mass-transfer and interaction of the gas stream with the atmosphere. Armed with all of this information, we could then model amplitude and phase changes for different nonradial modes during the primary eclipses (PSF-modelling). Using 3-D hydrodynamic simulations of mass transfer for target systems, we can compare hot gas structures in the circumbinary space found spectroscopically to those predicted by 3 -D hydrodynamic simulations. 
Table 1: A list of the binary systems with oEA components included in our survey. For each star, we give the spectral type (SpT), the orbital period $P_{\text {orb }}$ (in days), the main pulsation frequency $f_{\text {puls }}$ (in $\mathrm{d}^{-1}$ ), the brightness in the $V$-band (in mag), the spectrograph(s) used for the observations, and the detection of non-radial NRPs. The systems with detected high-degree NRPs are marked with "Yes" in the last column while the other systems are still being analysed.

\begin{tabular}{|l|llllll|}
\hline Star & SpT & $P_{\text {orb }}($ days $)$ & $f_{\text {puls }}\left(\mathrm{d}^{-1}\right)$ & V $(\mathrm{mag})$ & Spectrograph & NRPs \\
\hline Y Cam & A7 V & 3.305766 & 15.05 & 10.60 & MRES & \\
WY Cet & A2 V & 1.939755 & 13.20 & 9.28 & HRS & Yes \\
IV Cas & A3 V & 0.998506 & 38.20 & 11.34 & MRES & \\
SY Cen & A5 V & 6.631357 & 10.68 & 11.29 & HRS & \\
R CMa & F1 V & 1.135954 & 21.02 & 5.70 & MRES/HERMES & \\
TW Dra & A8 V & 2.806804 & 18.95 & 7.46 & TLS & Yes \\
TZ Dra & A7 V & 0.866028 & 29.00 & 9.33 & MRES & \\
AS Eri & A3 V & 2.664145 & 59.04 & 8.30 & MRES/HRS/HERMES & Yes \\
TZ Eri & A5 V & 2.606113 & 18.94 & 9.61 & MRES & \\
VY Hya & A8 V & 2.001180 & 28.44 & 9.64 & HRS & Yes \\
LT Her & A4 V & 1.084034 & 31.40 & 10.55 & MRES & \\
Y Leo & A3 V & 1.686109 & 34.48 & 10.07 & MRES & \\
RR Lep & A4 V & 0.915423 & 31.87 & 10.14 & MRES/HRS & \\
VY Mic & A4 III/IV & 4.436373 & 12.23 & 9.54 & HRS & \\
FO Ori & A3 V & 18.80054 & 34.25 & 9.48 & MRES & \\
V392 Ori & A5 V & 0.659282 & 40.56 & 10.67 & MRES & \\
IU Per & A4 V & 0.857020 & 42.10 & 10.56 & MRES & Yes \\
GQ Tra & A3 V & 2.339450 & 45.64 & 9.89 & HRS & Yes \\
VV UMa & A2 V & 0.687378 & 28.90 & 10.28 & MRES & Yes \\
BD Vir & A5 V & 2.548537 & 34.16 & 9.95 & HRS & \\
UW Vir & A2 V & 1.810776 & 28.78 & 9.13 & HRS & \\
KIC 11175495 & A4 V & 1.096477 & 64.46 & & MRES & \\
KIC 8553788 & A7 V & 1.606167 & 58.26 & 12.19 & MRES & \\
TYC 7053-566-1 & F0 V & 5.104238 & 13.45 & 11.51 & HRS & \\
\hline
\end{tabular}




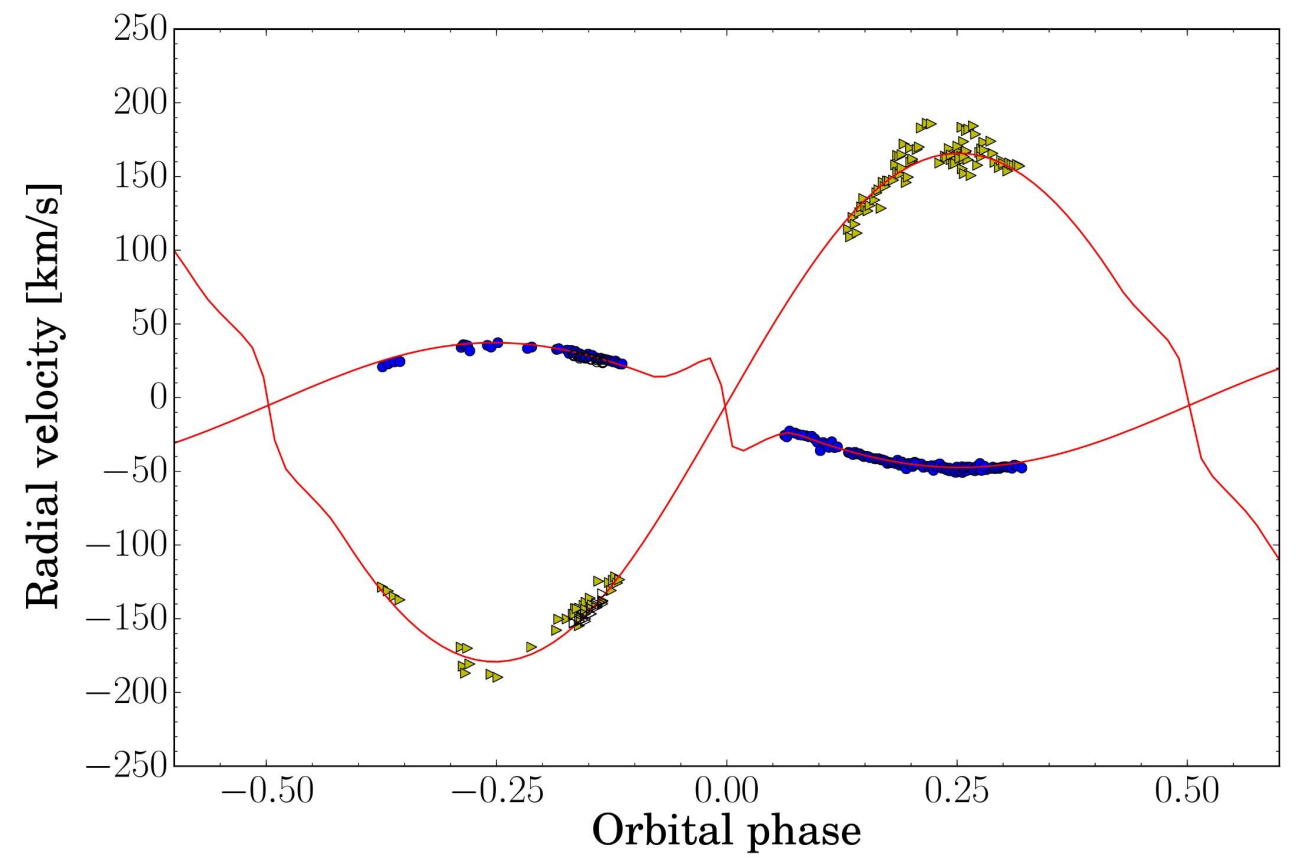

Figure 1: The orbits of the primary (blue circles) and secondary (green triangles) component of RX Hya.

\subsection{First results}

In Table 1, we listed oEA stars observed spectroscopically at the TNO, SAAO, TLS, and RMO. For the analysis of the spectral line profiles of the components, we used the Least-Squares Deconvolution (LSD) profiles constructed using the technique and the code described by Tkachenko et al. (2013). The radial velocities of each component were measured from the centers of gravity of the LSD profiles of the primary and the secondary components.

Fig. 1 shows the measured radial velocities and calculated orbits of the pulsating A-type and the Roche lobe filling K-type components of the RX Hya system. Fig. 2 shows the first discoveries of strong features runing from the blue to the red wings in the LSD profiles of spectral lines in AS Eri and VY Hya. Fig. 3 shows similar waves running from the blue to the red wings in the profiles of the primary component of GQTrA. These moving features are caused by high-degree $(\ell>4)$ non-radial pulsation modes running in the direction of rotation. This is a very typical picture of the excitation of low- and high-degree non-radial modes in rapidly-rotating oEA stars.

\section{Conclusion}

We present the first results of a spectroscopic survey of oEA stars. We report for the first time about the discovery of high-degree NRPs in the mass-accreting components of AS Eri, WY Cet, VY Hya, GQ TrA, BD Vir, and UW Vir. The spectroscopic survey will be continued for the new oEA stars discovered with the Transiting Exoplanet Survey Satellite (TESS; Ricker et al. 2015) and will be a part of the BINA collaboration. 

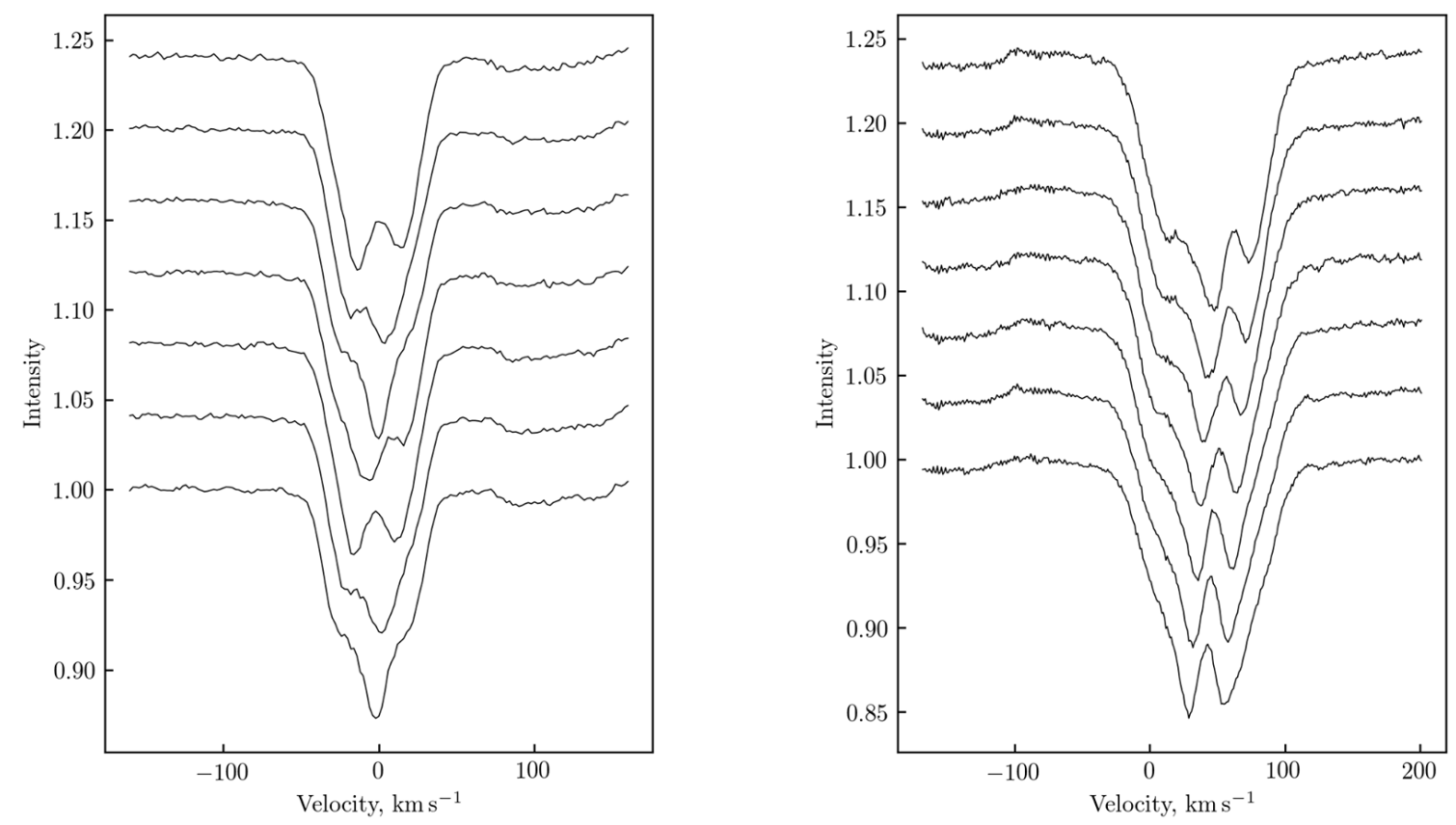

Figure 2: The time-series of LSD line profiles of the primary components of AS Eri (left panel) and VY Hya (right panel) showing line profile variations due to high-degree pulsations. The blue-to-red running features are caused by prograde non-radial modes. The time axis is from bottom to top.

\section{Acknowledgements}

This research is a part of the research activity supported by the National Astronomical Research Institute of Thailand (NARIT), Ministry of Science and Technology of Thailand. HL acknowledges support by the German DFG grant LE 1102/3-1.

\section{References}

Bramall D. G., Schmoll J., Tyas L. M. G. et al. 2012, Proc. SPIE, 8446, 84460A, 9

De Cat P., Surdej J., Omar A., De Becker M., Joshi S., 2018, BSRSL, 87, 1

Lehmann H., Mkrtichian D. E. 2004, A\&A, 413, 293

Lehmann H., Mkrtichian D. E. 2008, A\&A, 480, 247

Lehmann H., Tsymbal V., Pertermann F. et al. 2018, A\&A, 615, A131

Mkrtichian D. E., Kusakin D. E., Rodríguez E. et al. 2004, A\&A, 419, 1015

Mkrtichian D. E., Lehmann H., Rodrǵuez E. et al. 2018, MNRAS, 475, 4745

Raskin G., Van Winkel H., Hensberge H. et al. 2011, A\&A, 526, 69

Ricker G. R., Winn J. N., Venderspek R. et al. 2015, JATIS, 1, 014003

Tkachenko A., Lehmann H., Mkrtichian D. E. 2009, A\&A, 504, 991

Tkachenko A., Lehmann H., Mkrtichian D. E. 2010, AJ, 139, 1327

Tkachenko A., Van Reeth T., Tsymbal V. et al. 2013, A\&A, 560, A37 

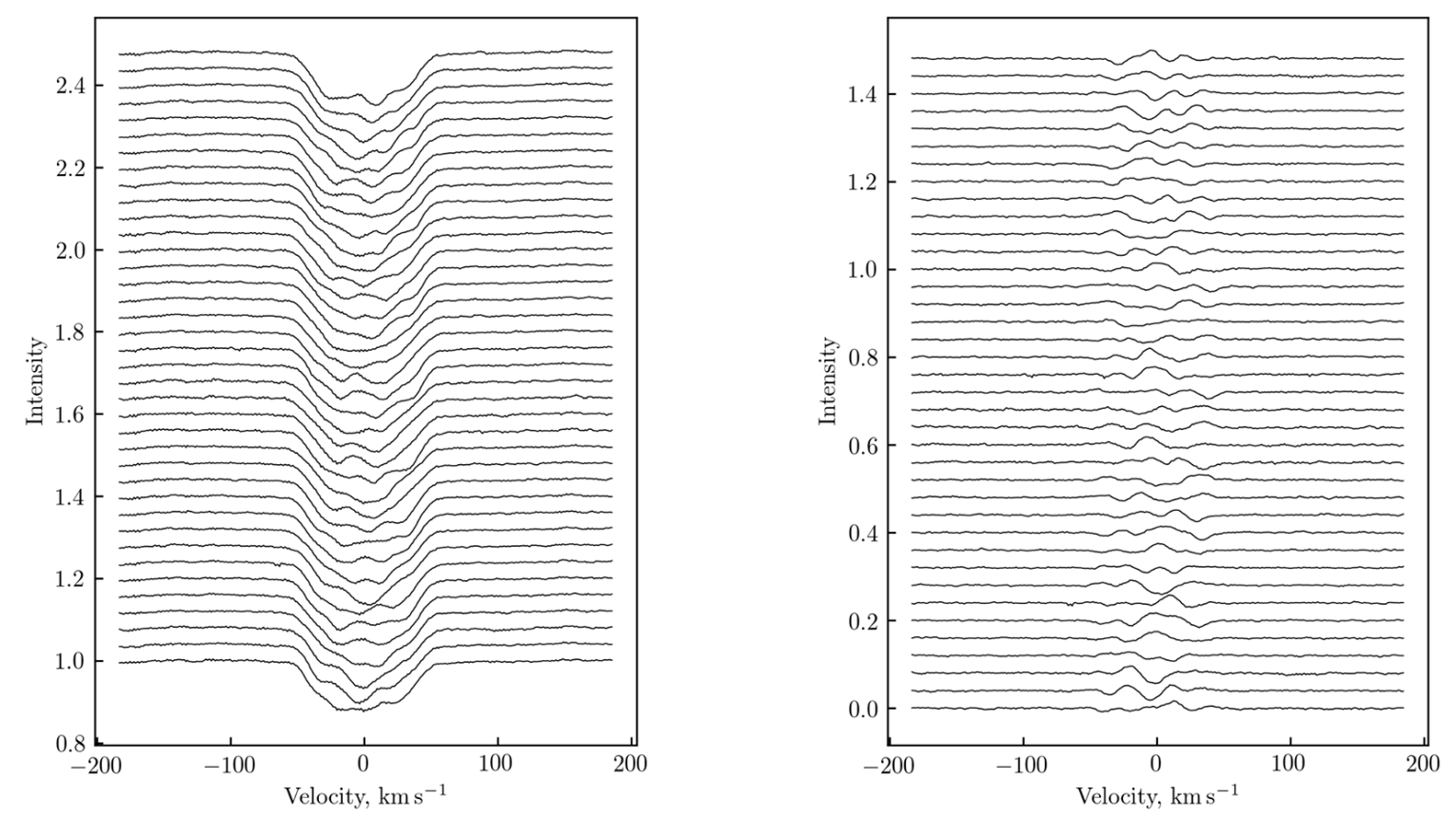

Figure 3: The time-series of LSD profiles of GQTrA (left panel) and residuals after removal of the mean profile (right panel). The time axis is from bottom to top. Bumps due to high-degree NRPs running across the line profiles are clearly visible. 